'Departamento de Filosofía, Universidad de Santiago de Chile (USACH). Santiago, Chile. ${ }^{2}$ Instituto de Ética e Historia de la Medicina, Facultad de Medicina, Eberhard Karls Universität Tübingen, Alemania. ${ }^{\mathrm{a} P h D}$.

Trabajo financiado por Proyecto DICYT 031953AS_DAS, "Envejecimiento saludable. Fundamentos normativos y filosóficos de la vejez", de la Universidad de Santiago de Chile, USACH y por Proyecto FONDECYT regular N¹210250, 2021-2024 (ANID). En ambos la Investigadora Responsable: Dra. Diana Aurenque.

La autora declara no tener conflictos de interés.

Recibido el 20 de octubre de 2020, aceptado el 12 de octubre de 2021

Correspondencia a: Dra. Diana Aurenque Universidad de Santiago de Chile (USACH). Departamento de Filosofía. Facultad de Humanidades.

Avda. Libertador Bernardo O'Higgins 3363 9170022 Estación Central, Chile. diana.aurenque@usach.cl

\section{¿Contradice la eutanasia o el suicidio asistido el ethos médico? Orientaciones para una ética médica moderna y pluralista}

\author{
DIANA AURENQUE ${ }^{1,2, a}$
}

\section{Is there a contradiction between medical ethos and euthanasia?}

\begin{abstract}
The practice of euthanasia in the medical context has a plurality of actors and interests, has ethical issues and the definition of "good death" is complicated. Therefore, it is necessary to be clear about the key concepts in the debate about this practice. But, above all we must ask ourselves to what extent euthanasia or assisted suicide contradicts the medical ethos in contemporary culture, aiming to evaluate the most acceptable medical and ethical options in our pluralistic societies. Only after such reflection and clarification, we will be able to understand the ethical conflicts of this practice and propose the appropriate ethical guidelines.

(Rev Med Chile 2021; 149: 1372-1376)
\end{abstract}

Key words: Euthanasia; Ethics, Medical; Suicide, Assisted.

\section{E} n Chile, se discute desde hace varios años y con diversa intensidad sobre la necesidad de disponer de una ley que permita y regule la práctica de la eutanasia-del griego eu-thanatos, palabra que semánticamente alude a un buen morir. Actualmente, no existe la posibilidad de optar a la eutanasia ${ }^{1}$. Uno de los casos recientes que más conmocionaron a la opinión pública nacional corresponde a la petición de la joven Valentina Maureira $^{2}$ de 14 años, quien le solicitó a quien, en ese tiempo era Presidenta de la República, Michelle Bachelet, le concediera la eutanasia para terminar con los sufrimientos ocasionados por la fibrosis quística que padecía. Pese a que dicha petición no fue (ni podía ser) concedida por falta de una legalidad idónea, Valentina falleció el 2015, su caso contribuyó enormemente a sensibilizar a la nación sobre la necesidad de una regulación que conceda a las personas la posibilidad de anticipar la muerte, con ayuda médica apropiada y en casos de sufrimientos extremos.

Al menos desde la secularización de la cultura, así como del reconocimiento de que las personas tenemos el derecho legítimo e inalienable de decidir con libertad sobre cómo conducir nuestras vidas, se ha constituido un sentido común con valores pluralistas. Esto implica aceptar que no existe una única forma de vivir bien -ni tampoco de morir bien- que ostente una fuerza normativa mayor y vinculante, ni para la humanidad ni para la praxis médica. En efecto, mientras que para algunos puede ser una "buena muerte" mantener hasta el final todos los recursos médico-tecnológicos para retrasar la llegada de la muerte; para otros, una "buena muerte" puede ser precisamente lo contrario, una muerte que interrumpa sufrimientos psíquicos o físicos irreversibles. Así, si pretendemos definir conceptualmente lo que constituye una "buena muerte" estamos condenados a una tarea tan imposible de satisfacer como constituir una buena ética ${ }^{3}$. Y a ello, se le adhiere otra dificultad: la palabra "eutanasia" en el ámbito médico y ético es especialmente equívoca y requiere de múltiples aclaraciones. En efecto, cuando segui- 
mos el debate nacional sobre eutanasia, debemos distinguirla en sus diversos sentidos -voluntaria/ involuntaria; activa/pasiva o directa/indirecta-, así como de otros conceptos con los que guarda relación, pero no una identificación.

Especialmente en el último tiempo, hemos visto que en nuestro país se ha actualizado un intenso debate público que invita a diversos actores-médicos ${ }^{\star}$, políticos, pacientes, bioeticistas, filósofos, abogados, entre otros- a tomar partido en la discusión. Dada la pluralidad de actores e intereses, así como a las dificultades propias de una determinación conceptual de aquello que puede ser una "buena muerte", sumado a la problemática ética de la práctica eutanásica en el contexto médico, es preciso no solo a) tener claridad sobre los conceptos claves en el debate, sino más bien y por sobre todo; b) preguntarnos hasta qué punto la eutanasia contradice el ethos médico en la cultura contemporánea, para con ello; c) evaluar las opciones médicas y éticas más aceptables en el contexto de sociedades pluralistas como la nuestra.

\section{Discusión y análisis}

En el debate médico actual la eutanasia refiere a la acción y decisión de una persona gravemente enferma o que sufre, respecto de optar libre y voluntariamente por acelerar el arribo de su muerte $^{4,5}$. Pero no todo adelantar la muerte ocurre de la misma manera ni significa lo mismo. La eutanasia activa también llamada directa o voluntaria, constituye el "acto médico de terminar con la vida de un paciente, bajo la voluntad

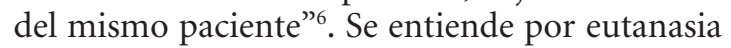
involuntaria cuando dicha determinación ocurre sin el consentimiento del paciente (por ej. cuando no puede consentir). La eutanasia pasiva remite al "acto médico de retirar, suspender o no iniciar un tratamiento que se limita a prolongar la vida de un paciente que se encuentra en situación de enfermedad terminal o irreversible" lo central es determinar que el proceso de muerte ya ha comenzado. Adicionalmente, debemos distinguir la eutanasia en las formas descritas del suicidio asistido. Este refiere a que, quien opta por

\footnotetext{
${ }^{\star}$ Por razones de extensión, usaremos el genérico masculino, pero se subentiende que aludimos a un horizonte genérico diverso.
}

acelerar su muerte y ejecuta por sí mismo la acción suicida -por ej. mediante la ingesta de un medicamento letal-, pero donde dicha dosis ha sido preparada por un tercero ${ }^{8}$. Finalmente, hay que distinguir estos últimos de la Limitación del esfuerzo terapéutico (LET) que refiere a la restricción $\mathrm{u}$ omisión de tratamientos terapéuticos en pacientes para aminorar sufrimiento, y que puede conducir a la muerte prematura del afectado. Pese a que algunos ven a la LET como sinónimo de eutanasia pasiva $^{9}$, nos inclinamos por la posición que no la considera como tal ${ }^{10}$ debido a que su propósito no es adelantar voluntariamente el momento de la muerte, sino que esto último es una consecuencia que se acepta, pero no se pretende ${ }^{11}$.

En medicina, el conflicto ético específico -no la pregunta filosófica por la justificación racional del suicidio ${ }^{12}$ - que trae consigo la posibilidad de legitimar y regular la eutanasia se relaciona principalmente con la pregunta por su permisibilidad como una práctica legítima y propia de la medicina. ¿A qué se debe la especificidad del conflicto? Esto lo leemos al menos desde dos tradiciones: una médica y otra histórico-metafísica. Esta última tiene que ver con la idea de que la muerte constituye un mal y que la vida es un bien en sí mismo. En la actualidad existe una larga tradición en filosofía moral que ha puesto en duda dicha opinión ${ }^{13}$. El argumento médico, sin embargo, es otro, aunque indirectamente relacionado.

Como es conocido, la medicina tiene como uno de sus pilares éticos fundamentales el primum non nocere. Este prohíbe a los médicos efectuar acciones que dañen a sus pacientes. En cuanto la medicina históricamente ha intentado promover la salud, evitar la enfermedad y con así, prolongar la vida, la búsqueda anticipada de la muerte parece contradecir uno de sus grandes principios, es decir, su propio ethos médico. Esto se agudiza en un contexto que asuma que la vida es un bien en sí mismo -lo que sostienen algunos adherentes de la idea de la "santidad de la vida" (argumento que se funda en consideraciones metafísico-teológicas). Desde aquí se comprende que la Asociación Médica Mundial (AMM) señale: "La eutanasia, es decir, el acto deliberado de poner fin a la vida de un paciente, aunque sea por voluntad propia o a petición de sus familiares, es contraria a la ética. Ello no impide al médico respetar el deseo del paciente de dejar que el proceso natural de la muerte siga su curso en la fase terminal de su 
enfermedad"14. En dicha declaración, la AMM rechaza enfáticamente a la eutanasia como parte de su práctica médica.

Pero también donde la eutanasia o el suicidio asistido han sido permitidos conviven apreciaciones distintas respecto de su relación con el ethos médico. Baste para ello analizar las diversas legislaciones internacionales así como asociaciones médicas en países como Holanda, Bélgica, Luxemburgo, Suiza, Alemania, Colombia y algunos estados de Estados Unidos como Oregon o Washington, que han regulado y permitido estas medidas. Actualmente otros países -además de Chile, Perú, España, Francia, Australia y el Reino Unido- también discuten sobre incluir legislaciones en estas materias. Desde el punto de vista ético, el debate se centra justamente en definir el rol de esta práctica en el contexto de la tarea médica. En Estados Unidos The American Medical Association's Council on Ethical and Judicial Affairs define a la eutanasia como "the administration of a lethal agent by another person to a patient for the purpose of relieving the patient's intolerable and incurable suffering"; $y$ aconseja que "instead of engaging in euthanasia, physicians must aggressively respond to the needs of patients at the end of life." Con ello, nos indican que rechazan la práctica activa de eutanasia, pero al mismo tiempo, se contradicen al sostener que los médicos" (b) (m) ust respect patient autonomy"15. ¿Cómo puede respetarse la autonomía de los pacientes si estos deciden adelantar su muerte y los médicos "agresivamente" no lo aceptan? Desde luego, esto implica que la eutanasia no es contemplada por toda la comunidad médica estadounidense como parte de su hacer, pero por otro lado, otras fracciones apoyan su participación en el suicidio asistido -en 2008 Washington lo legalizó con 60\% de los votos ${ }^{16}$ y en Oregón cuenta con un apoyo del $51 \%$ de los médicos generalistas, $48 \%$ enfermeras de hospicio, entre otros ${ }^{17}$. Otro ejemplo: En Alemania, la Bundesärztekammer (Asociación Médica Alemana) tras debates agitados reformuló sus principios acerca de la asistencia médica de la persona moribunda. Mientras en el 2004 ésta decía que "(l)a participación del médico contradice el ethos médico y puede ser punible", en 2011 fue modificado a "(l)a participación del médico en el suicidio no es una tarea médica"18. Si bien la modificación rechaza explícitamente que la eutanasia contraríe el ethos médico, para sus críticos, dicha modificación deja a la eutanasia fuera de sus labores, lo que sería aún más preocupante ${ }^{19}$. En la medida de que los médicos son los especialistas par excellance de aquello que tiene que ver con la vida y su promoción, también saber sobre su fin es otra cara posible de sus labores e incluso responsabilidades éticas ${ }^{20}$. En Holanda, que cuenta con eutanasia y suicidio asistido, relaciona y justifica estas prácticas principamente con el deber ético del médico de aliviar el sufrimiento de sus pacientes ${ }^{21}$.

Responder a la pregunta de si la eutanasia o el suicidio asistido contradicen el ethos médico adecuadamente exige tener claridad sobre las metas de la medicina contemporánea ${ }^{22}$. Si bien aquel debate sigue en desarrollo, permite al menos distinguir entre dos posibles aproximaciones: o bien orientarnos por un planteamiento teleológico (según el cual la medicina tendría metas inherentes y fieles al mandato hipocrático); o bien por uno consensual (que sostenga que sus tareas cambian en función de acuerdos y a procesos deliberativos). En la medida de que es evidente que la práctica médica ha sufrido variaciones a lo largo de la historia, acorde a avances epistemológicos, científicos, así como también valórico-culturales, resulta difícil defender una postura teleológica. Precisamente la inclusión del principio de respeto a la autonomía de los pacientes como un mandato ético fundamental, ausente en el código hipocrático, demuestra que la medicina reformula y se replantea sus límites y posibilidades a partir de nuevos consensos (lo que tampoco implica que obtenga validez absoluta). A raíz de los desafíos emergentes que trae consigo la medicina contemporánea y altamente tecnificada, el Hasting Center (1996) propone cuatro metas fundamentales que justamente intenta propiciar nuevos acuerdos en la comunidad médica internacional: 1) la prevención de enfermedad y daños y la promoción y mantenimiento de la salud; 2) el alivio del dolor y del sufrimiento causado por enfermedades; 3 ) el cuidado y la cura de aquellos que están enfermos y el cuidado de aquellos que no pueden ser curados y 4) evitar la muerte prematura y la búsqueda de una muerte pacífica ${ }^{23}$. Evidentemente, estas metas no son exhaustivas. Sin embargo, permiten ofrecer orientaciones generales para afrontar nuevos retos de la praxis clínica. Para nuestro análisis, el punto 4 , si bien indica la importancia de "evitar la muerte prematura", al mismo tiempo comanda al médico a "la búsqueda de una muerte pacífica." Este 
último principio, bien puede incluir la eutanasia o el suicidio asistido como opciones, autodeterminadas por los pacientes, como formas de alcanzar una "muerte pacífica” o también llamada "digna".

Como en la mayoría de los conflictos ético-médicos, también el cuestionamiento ético sobre la permisibilidad de la eutanasia exige poner al descubierto el choque de valores o principios en juego. Por un lado, el deber de cuidar la vida (como rol del ethos médico), y por otro, encontramos dos principios éticos que se le oponen: el deber del médico de aliviar el sufrimiento de sus pacientes y el deber de respetar su autonomía. En este sentido, debemos aceptar que la eutanasia contradice al ethos médico si éste último significa evitar a toda costa la muerte; sin embargo, es igualmente cierto que no contradice al ethos médico, si le atribuimos la tarea de aliviar el sufrimiento de sus pacientes y si coincidimos que la muerte no siempre es un mal. Y, si a dichos argumentos, se le suma el deber ético más moderno, pero absolutamente inviolable en la ética médica de respetar la autonomía de las personas, encontramos dos principios éticos que permiten ver a la eutanasia como aceptable desde el punto de vista ético. Como indica un estudio sistemático, en las legislaciones actuales la argumentación ético-médica para permitir la inclusión de eutanasia y también del suicidio asistido consiste precisamente en relevar la importancia de, por un lado, respetar la autonomía de las personas $y$, por otros, de respetar sus obligaciones de actuar en beneficio de sus pacientes (aliviando su sufrimiento $)^{24}$.

Si lo anterior es correcto, para la legitimidad de prácticas eutanásicas o de asistencia al morir en el contexto médico, deben existir criterios claros e indiscutibles para aceptarla. Ellos deben dar prueba fehaciente de que lo que está en juego es velar porque se respeten justamente los principios mandatorios: beneficiar al paciente mediante la supresión de su sufrimiento y respetar su autonomía. De aquí se desprende que los criterios de una legislación apropiada deben ser detallados y verificados cuidadosamente. Entre ellos destacan los siguientes: 1) el requerimiento voluntario del paciente; 2) la constatación de un sufrimiento físico o psicológico insoportable e irreversible, y que no puede ser aliviado con sedaciones paliativas ni tampoco remite a un estado depresivo circunstancial; 3) el pleno conocimiento de su situación y pronóstico inmejorable e incurable,
4) la autodeterminación de inviabilidad de otras opciones; 5) la confirmación de este diagnóstico por dos médicos independientes $y, 6$ ) que el término de la vida sea realizado lege artis.

\section{Conclusión}

La eutanasia así como el suicidio asistido representan nuevos desafíos para la medicina contemporánea y la auto comprensión que tenga de su ethos y tareas fundamentales. En cuanto ni la medicina ni la filosofía ni ideología alguna puede ofrecer una interpretación unívoca con validez normativa para determinar aquello que constituye una "buena muerte", las legislaciones deben proporcionar un marco regulatorio general e inclusivo que, si bien proteja a la ciudadanía de amenazas externas, también las respalde en sus decisiones particulares y personalísimas.

La medicina contemporánea ha sabido y debe siempre seguir revisando su ethos propio, sus tareas fundamentales y desafíos de acuerdo, no solo a las necesidades cambiantes de la sociedad, la cultura y las posibilidades tecnológicas de turno, sino también acorde a principios éticos. Si una legislación aprueba la eutanasia o el suicidio asistido, lo hace en ambos casos con el fin de otorgar protección y asistencia a personas en casos extremos, de sufrimiento intolerable e irrecuperable - no en delirios suicidas transitorios. Por ello, los médicos deben tener la tranquilidad de que no dejan de cumplir su tarea por apresurar la muerte en estos casos, sino más bien, se mantienen fieles a un ethos que intenta aliviar al que sufre, y permitirle morir en las mejores condiciones posibles, devolviéndoles un poco de decisión vital -dignidad llaman algunos-, ahí, donde precisamente sus condiciones vitales son inmejorables.

Agradecimientos: Para asuntos de edición del presente texto agradezco a Sofía Aguirre Margery.

\section{Referencias}

1. Carrasco V, Crispi M. Eutanasia en Chile: una discusión pendiente Rev Med Chile 2016; 144: 1598-604.

2. Eutanasia en Chile: una discusión pendiente. Rev Med Chile 2016; 144: 1598-604.

3. Wiesing U. Die Ethik und ihre Herausforderungen in 
einer pluralistischen Gesellschaft, en: Borasio, Gian Domenico/ Ralf, J. Jox/ Taupitz, Jochen/ Wiesing, Urban, (ed.), Assistierter Suizid: Der Stand der Wissenschaft. Mit einem Kommentar zum neuen Sterbehilfe-Gesetz, Springer Verlag, Berlin/Heidelberg 2017; 87-9.

4. Thiele, Felix, Sterbehilfe, Bioethische Themen en: Sturma, Dieter/Heinrichs, Bert (edit), Handbuch Bioethik. 2015; 394-400, trad. D.A.

5. Borasio G, Jox R, Jochen Taupitz, Urban Wiesing, Selbstbestimmung im Sterben - Fürsorge zum Leben, Stuttgart 2014.

6. Carrasco V, Crispi M. Eutanasia en Chile: una discusión pendiente. Rev Med Chile 2016; 144: 1598-604.

7. Carrasco V, Crispi M. Eutanasia en Chile: una discusión pendiente. Rev Med Chile 2016; 144: 1598-604.

8. Borasio G, Jox R, Jochen Taupitz, Urban Wiesing, Selbstbestimmung im Sterben - Fürsorge zum Leben, Stuttgart 2014.

9. Carrasco V, Crispi M. Eutanasia en Chile: una discusión pendiente. Rev Med Chile 2016; 144: 1598-604.

10. Goic, A. Apuntes sobre la Eutanasia. Rev Med Chile 2005; 133: 371-5.

11. Beca JP, Ortiz A, Solar S. Derecho a Morir. Rev Med Chile 2005; 133: 601.

12. Ugarte JJ. El derecho a la vida y la Constitución. Rev Chil Derecho 2006; 33: 509-27.

13. Rodríguez E. La eutanasia y sus argumentos. Ars Medica 2000; 2: 45-57.

14. Borasio G, Jox R, Jochen Taupitz, Urban Wiesing, Selbstbestimmung im Sterben - Fürsorge zum Leben, Stuttgart 2014.

15. Schramme T, Edwards S (ed.), Handbook of the philosophy of medicine, Vol 1, Springer Dordrecht 2017; 207-22.

16. Taylor, S. James, en: Schramme T, Edwards S (ed.), Handbook of the philosophy of medicine, Vol 1, Springer Dordrecht, 2017; 529-38.

17. Asociación Médica Mundial, Declaración de la AMM sobre la eutanasia, 2017. Disponible en: https://www. wma.net/es/policies-post/declaracion-de-la-amm-sobre-la-eutanasia/
18. American Medical Association, Euthanasia, Code of Medical Ethics Opinion 5.8. Disponible en: https://www. ama-assn.org/delivering-care/ethics/euthanasia

19. Vizcarrondo F, Euthanasia and Assisted Suicide: The Physician's Role, Linacre Q. 2013 May; 80 (2): 99-102.

20. Ganzini L, Legalized Physician Assited Death in Oregon -Eighteen Years` Experience, 7-20, En: Borasio, Gian Domenico/ Ralf, J. Jox/ Taupitz, Jochen/ Wiesing, Urban, (ed.), Assistierter Suizid: Der Stand der Wissenschaft. Mit einem Kommentar zum neuen Sterbehilfe-Gesetz, Springer Verlag, Berlin/Heidelberg 2017.

21. Bundesärztekammer, Grundsätze der Bundesärztekammer zur ärztlichen Sterbebegleitung, 2011. Disponible en: https://www.bundesaerztekammer.de/fileadmin/ user_upload/downloads/Sterbebegleitung_17022011.pdf

22. Borasio G, Jox R, Jochen Taupitz, Urban Wiesing, Selbstbestimmung im Sterben - Fürsorge zum Leben, Stuttgart 2014.

23. Thiele, Felix, Sterbehilfe, Bioethische Themen en: Sturma, Dieter / Heinrichs, Bert (edit), Handbuch Bioethik 2015; 394-400, trad. D.A.

24. Buiting $\mathrm{H}$, van Delden J, Onwuteaka-Philpsen B, Rietjens J, Rurup M, van Tol D, Gevers J, van der Maas P, van der Heide A, Reporting of euthanasia and physician-assisted suicide in the Netherlands: descriptive study, BMC Med Ethics. 2009 Oct 27; 10: 18. doi: 10.1186/1472-6939-10-18. Disponible en: https://www. ncbi.nlm.nih.gov/pmc/articles/PMC2781018/

25. Schramme T, Edwards S (ed.), Handbook of the philosophy of medicine, Vol.1, Springer Dordrecht 2017.

26. Hastings Center, The goals of medicine. Setting New Priorities. Special Suplement, Nov-Dic 1996. Disponible en: https://onlinelibrary.wiley.com/doi/abs/10.1002/ j.1552-146X.1996.tb04777.x

27. Buiting $H$, van Delden J, Onwuteaka-Philpsen B, Rietjens J, Rurup M, van Tol D, Gevers J, van der Maas P, van der Heide A, Reporting of euthanasia and physician-assisted suicide in the Netherlands: descriptive study, BMC Med Ethics. 2009 Oct 27; 10: 18. doi: 10.1186/1472-6939-10-18. Disponible en: https://www. ncbi.nlm.nih.gov/pmc/articles/PMC2781018/ 